\title{
Preissenkung gegen Pillen-Splitting
}

- Das Unternehmen Bayer Vital hat am

1. Mai den Apothekenabverkaufspreis für die 10-mg- und 5-mg-Dosierungen des PDE5 -Inhibitors Vardenafil (Levitra ${ }^{\circledR}$ ) um etwa $50 \%$ beziehungsweise $60 \%$ gesenkt. Die Kosten pro 10-mg-Tablette sinken von rund 12 EUR auf 6 EUR.

Hintergrund für diese Maßnahme sind die Ergebnisse einer großen Marktstudie, die zum Einsatz von PDE-5-Inhibitoren durchgeführt wurde. Die Studie zeigte, dass der Kostenfaktor für Patienten mit erektiler Dysfunktion eine entscheidende Bedeutung hat, da sie in der Regel die Therapie aus eigener Tasche zahlen müssen. Um Kosten zu sparen, nehmen viele Patienten derzeit die Therapie nicht oder nicht in vollem Umfang wahr. $43 \%$ gaben an, ihr Sexualleben genau zu planen, um keine Pillen zu „vergeuden“. Kostengründe sind auch dafür verantwortlich, dass mindestens $50 \%$ der Patienten, die PDE-5-Hemmer einnehmen, die Pillen zerteilen. Ein problematisches Vorgehen, denn die Tabletten sind nicht für eine Teilung bestimmt und eine exakte Dosierung daher nicht gewährleistet. Darüber hinaus suchen Patienten im Internet nach preiswerten Alternativen und laufen dabei Gefahr, Fälschern aufzusitzen und ihre Gesundheit durch Pillen aus dubiosen Internet-Käufen zu gefährden.

„Die Therapie der erektilen Dysfunktion hat eine enorme Bedeutung für die Gesundheit und Lebensqualität von Männern im besten Alter", erklärt Prof. Frank Sommer, Universitätsklinikum Hamburg-Eppendorf. „Deshalb sind Maßnahmen, die mehr Männern mit Potenzstörungen eine fälschungssichere und wirksame Therapie ermöglichen, grundsätzlich zu begrüßen.“

Nach Informationen von

Bayer Vital, Leverkusen

\section{Sekundärer Hyperparathyreoidismus - eine Domäne von Kalzimimetika}

\begin{abstract}
_ Patienten mit fortgeschrittener Niereninsuffizienz entwickeln häufig einen sekundären Hyperparathyreoidismus (SHPT), der vor allem wegen der damit assoziierten Gefäßkalzifizierung und der kardiovaskulären und ossären Komplikationen eine therapeutische Herausforderung ist. Die Progression der Koronarkalzifizierung kann neuesten Daten zufolge verlangsamt werden, wenn die Patienten zusätzlich zur Standardtherapie (Vitamin D plus Phosphatbinder) das Kalzimimetikum Cinacalcet (Mimpara ${ }^{\circledR}$ ) erhalten, berichtete Prof. Paolo Raggi, Atlanta/USA. Das haben die Ergebnisse der ADVANCE-Studie (a randomized vascular calcification study to evaluate the effects of cinacalcet) gezeigt, eine prospektive, randomisierte, kontrollierte Phase-IV-Studie. An der Studie nahmen 360 Dialysepatienten mit SHPT teil. Sie erhielten jeweils zur Hälfte entweder Cinacalcet (30-180 $\mathrm{mg} / \mathrm{d}$ ) und niedrig dosiertes Vitamin D oder im anderen Studienarm Vitamin D in flexibler Dosierung als Kontrolle. Primärer Endpunkt war die mit dem AgatstonScore ermittelte Veränderung der koronar-
\end{abstract}

arteriellen Kalzifikation (CAC) nach 52 Wochen im Vergleich zum Baseline-Wert.

Nach 52 Wochen wurde laut Raggi in der Verumgruppe ein geringerer Anstieg der Kalzifizierung beobachtet als in der Kontrollgruppe. Der Agaston-Score stieg unter Cinacalcet plus niedrig dosiertem Vitamin D um $24 \%$ und bei einer alleinigen Therapie mit flexibel dosiertem Vitamin D um 31\%, wobei die statistische Signifikanz knapp verfehlt wurde $(p=0,073)$.

Die derzeit noch laufende placebokontrollierte Phase-III-Studie EVOLVE (evaluation of cinacalcet $\mathrm{HCl}$ therapy to lower cardiovascular events) untersucht bei $3.800 \mathrm{~Pa}$ tienten, ob Cinacalcet das Risiko der allgemeinen Mortalität und/oder von nicht tödlichen kardiovaskulären Ereignissen verringert. Die Ergebnisse werden im Jahr 2011 erwartet.

aam

Pressekonferenz „Pioneering science in chronic kidney disease management " im Rahmen des ERA-EDTA-Kongresses, München, 25. Juni 2010. Veranstalter: Amgen, München

\section{Senföle statt Antibiotika}

Eine Antibiotikatherapie bei Harnwegsinfekten (HWI) hat Nachteile: Die Vaginalmykose ist eine der häufigsten Nebenwirkungen. Ein weiteres Problem vor allem bei Langzeittherapien - ist die Entwicklung von Resistenzen. Weder Scheidenpilz noch Antibiotikaresis tenzen treten dagegen bei der Behandlung unkomplizierter HWI mit Senfölen auf. Senföle sind sekundäre Pflanzenstoffe, die Pflanzen zu ihrem eigenen Schutz produzieren. Verschiedene In-vitro-Studien am Universitätsklinikum Freiburg bescheinigen den Senfölglykosiden aus Kapuzinerkressenkraut und Meerrettichwurzel (ANGOCIN ${ }^{\circledR}$ Anti-Infekt N) ein breites antibakterielles Wirkspektrum. Die aktuellste Untersuchung zum Thema zeigt, dass die Senfölglykoside aus ANGOCIN ${ }^{\circledR}$ Anti-Infekt $\mathrm{N}$ auch bei Nachweis von resistenten oder multiresistenten Erregern eine Behandlungsoption sein können. Aufgrund ihrer guten Verträglichkeit sind Senföle zudem zur Prophylaxe geeignet.

Nach Informationen von Repha, Langenhagen

\section{Alternative zu Morphin}

Menschen mit starken Schmerzen können jetzt auf eine nebenwirkungsarme und kostendämpfende Alternative zu Morphin zurückgreifen: Das Unternehmen STADA hat das Generikum Hydromorphon STADA ${ }^{\circledR}$ Retardtabletten in den Wirkstärken $4 \mathrm{mg}, 8 \mathrm{mg}, 16 \mathrm{mg}$ und $24 \mathrm{mg}$ auf den Markt gebracht. Opioidtypische $\mathrm{Ne}$ benwirkungen wie Müdigkeit, Übelkeit und Obstipation sind im Vergleich zu Opioiden der WHO-Stufe 2 gering. Die niedrige Plasma-Eiweißbindung und der geringe Einfluss auf das Cytochrom-P450-System stehen zudem für eine niedrige Wechselwirkungsrate mit anderen Medikamenten. Die analgetische Wirkung des Opioids Hydromorphon ist etwa fünf bis siebeneinhalb mal stärker als die von Morphin. Mit einer Wirkdauer von circa zwölf Stunden sind Hydromorphon STADA ${ }^{\circledR}$ Retardtabletten für die regelmäßige, zweimal tägliche Gabe bei starken Schmerzen geeignet.

Nach Informationen von Stada, Bad Vilbel 